\title{
Exercise resistance decoded
}

Even though the beneficial effects of exercise on health are well known, not everybody derives similar benefits from training in terms of endurance capacity and metabolic health.

In a new study published in Nature Medicine, Hirofumi Misu and colleagues show that the hepatokine selenoprotein $\mathrm{P}$ mediates exercise resistance in mice via its receptor in muscle (low-density lipoprotein receptor-related protein 1; LRP1). The finding that an endogenous liver-secreted factor inhibits the effects of exercise contrasts with the previously held notion that exercise resistance is determined at or before birth.

Mice globally deficient in selenoprotein $\mathrm{P}$ or with muscle-specific knockout of Lrp1 $\left(L_{r p} 1^{-/}\right)$fed a high-fat diet for 1 month (to induce obesity) and then subjected to exercise training (treadmill; 30 min per day for 5 weeks) exhibited greater aerobic exercise capacity in endurance tests than did trained wild-type mice. The 'super-endurance' phenotype of both knockout mice was accompanied by increased phosphorylation of AMPK and expression of Ppargc1a (which encodes PGC $1 \alpha$ ) in skeletal muscle, which were mediated by increased production of reactive oxygen species. Conversely, AMPK phosphorylation and Ppargc1a expression induced by a single bout of exercise was suppressed in $\mathrm{Lrp}^{-1}$ mice pretreated with selenoprotein P. Importantly, in a cohort of 31 sedentary, postmenopausal women without obesity or type 2 diabetes mellitus who underwent aerobic exercise training (walking and cycling; 3 days per week for 8 weeks), increased pre-training plasma levels of selenoprotein $\mathrm{P}$ were shown to be predictive of the ineffectiveness of training on endurance capacity.

While highlighting the therapeutic potential of targeting the selenoprotein P-LRP1 axis for treating metabolic diseases associated with a sedentary lifestyle, the findings also raise the possibility that selenoprotein $P$ could modulate exercise responsiveness in situations other than overnutrition-related conditions, such as ageing-related exercise resistance.

David Holmes

ORIGINAL ARTICLE Misu, H. et al. Deficiency of the hepatokine selenoprotein $\mathrm{P}$ increases responsiveness to exercise in mice through upregulation of reactive oxygen species and AMPactivated protein kinase in muscle. Nat. Med. http://dx.doi.org/10.1038/nm.4295 (2017) 